\section{Are renoprotection guidelines followed in elderly patients with diabetes?}

US guidelines recommend that diabetic patients with nephropathy, hypertension, or both, use angiotension-converting-enzyme (ACE) inhibitors or angiotension-II receptor blockers (ARBs) to help slow progression of chronic kidney disease. Winkelmayer et al. used Medicare data to explore application of these guidelines to treatment of elderly diabetic patients ( $\geq 65$ years) who were eligible to receive comprehensive drug treatment from the state of Pennsylvania in 2002. Patients with end-stage renal disease were excluded from the analysis.

The primary outcome was the filling of a prescription for either an ARB or an ACE inhibitor during January-March 2003. Of the 30,750 eligible diabetic patients (mean age $78.4 \pm 6.4$ years, $81 \%$ female), 21,138 had received a diagnosis of hypertension, nephropathy, or both. Although these patients were eligible for comprehensive drug treatment with minimal copayments, only $50.7 \%$ of diabetic patients with hypertension and/or proteinuria filled a prescription for an ACE inhibitor or ARB during the 3 -month period. Older patients (75-84 years and $\geq 85$ years) were significantly less likely to fill a prescription for the drugs than patients aged $65-74$ years $(P=0.01$ and $P<0.001$, respectively). Patients with proteinuria but not hypertension were less likely to fill a prescription for the drugs than patients with hypertension only $(P=0.03)$.

The authors conclude that renoprotection guidelines were not being followed in the elderly diabetic patients they studied, but acknowledge that the study did not assess whether this was because drugs were not prescribed by physicians or because prescriptions were not filled by patients.

Rebecca Ireland

Original article Winkelmayer WC et al. (2005) Underuse of ACE inhibitors and angiotensin II receptor blockers in elderly patients with diabetes. Am J Kidney Dis 46: 1080-1087

\section{Do ACE inhibitors and ARBs have specific renoprotective effects?}

In addition to their capacity to lower systemic blood pressure, angiotensin-converting-enzyme (ACE) inhibitors and angiotensin-II receptor blockers (ARBs) are thought to have specific renoprotective effects in patients with diabetic and nondiabetic nephropathy. Some guidelines advocating the use of these drugs in renal disease, however, base their recommendations on the results of placebo-controlled trials with no clinically relevant endpoints.

Casas et al. have therefore investigated the effects of ACE inhibitors and ARBs on the progression of renal disease, by undertaking a meta-analysis of 127 randomized, controlled trials that used either placebos or other antihypertensive drugs as comparators. Patient outcomes were compared using the primary endpoints of a doubling of the baseline serum creatinine concentration and occurrence of end-stage renal disease. Albuminuria levels, serum creatinine levels and glomerular filtration rates were also analyzed as secondary markers of kidney function.

When ACE inhibitors and ARBs were compared with other antihypertensive drugs, they were shown to slightly reduce the incidence of end-stage renal disease in patients with nondiabetic nephropathy, but this beneficial effect was less evident in large studies with $\geq 500$ participants. In patients with diabetic nephropathy, however, ACE inhibitors and ARBs did not slow the progression of renal disease more effectively than other antihypertensive drugs. As it is still not clear whether there are any specific beneficial effects of ACE inhibitors and ARBs on renal function, the authors note that there is little evidence to support their use for renoprotection.

Claire Braybrook

Original article Casas JP et al. (2005) Effects of inhibitors of the renin-angiotensin system and other antihypertensive drugs on renal outcomes: systematic review and meta-analysis. Lancet 366: 2026-2033

\section{Sonography is not a suitable method for assessing short-term disease progression in ADPKD}

Recently published results from the Consortium of Renal Imaging Studies in Polycystic Kidney Disease (CRISP) indicate that ultrasonography lacks the accuracy and precision needed to follow short-term progression in patients with autosomal dominant polycystic kidney disease (ADPKD).

ADPKD patients from four different centers underwent ultrasonography and MRI at 